\title{
Multi-Group Frequency Hopping OFDMA Based on Statistical Multiplexing
}

\author{
Xinghui Su, Tiejun Lv, Member, IEEE, Ping Gong, Xiang Yun and Shaoshi Yang \\ Department of Information Engineering \\ Beijing University of Posts and Telecommunications (BUPT) \\ Beijing, China \\ xhui.su@gmail.com, lvtiejun@tsinghua.org.cn
}

\begin{abstract}
In this paper, the multi-group frequency hopping OFDMA (MG-FH OFDMA) based on the statistical multiplexing is proposed for the downlink cellular system. Compared with the existed random frequency hopping OFDMA (RFH-OFDMA) system utilizing the statistical multiplexing, the proposed MG-FH OFDMA invokes the deterministic hopping pattern to reduce the number of subcarrier collisions. By dividing all users into different groups, the subcarriers are utilized sufficiently. Latin Square hopping pattern and user index updating scheme are applied to randomize the subcarrier collisions among users. The user capacity, defined as the maximum number of users served with a basic data-rate in a cell, is calculated with the consideration of intra-cell capacity and the other cell interference (OCI). Results show that the proposed MG-FH OFDMA achieves higher user capacity than that of the RFH-OFDMA.
\end{abstract}

Keywords-Orthogonal Frequency Division Multiple Access (OFDMA); frequency hopping(FH); statistical multiplexing; user capacity

\section{INTRODUCTION}

Orthogonal Frequency Division Multiplexing (OFDM) is an effective multi-carrier solution for broadband wireless communication. In the OFDM system, the data stream with high rate is transformed into a number of lower rate components. Then, the lower rate components are mapped onto different subcarriers which are orthogonal in frequency domain. Since the bandwidth of each subcarrier is narrower than the coherent bandwidth of the propagation channel, the frequency-selective fading is converted into the flat fading [1].

Orthogonal frequency division multiple access (OFDMA) is a multi-user version of the OFDM digital modulation scheme. Multiple accesses are achieved in OFDMA by assigning subsets of subcarriers to individual users [1]. This simultaneously allows low data rate transmission from several users. Combining OFDMA with frequency hopping (FH) technology, what forms FH-OFDMA can eliminate the narrow band and partial-band interference. Deterministic frequency hopping OFDMA assigns the subcarriers to users according to the predetermined hopping patterns over time. It exploits frequency diversity by appropriately choosing the FH pattern [3], [4].

In the modern communication system, data service is becoming the dominant traffic. Electronic media, such as email, E-ZIN and web browsing, will be the main data traffic in wireless communication system. This data traffic appears to be inherently bursty and generally exhibits some user activity. Based on the new characteristic of the communication system, statistical multiplexing technology has attracted attentions of the researchers. Statistical multiplexing is a method in which users only occupy a given link when they send packets. It allows users to select the same subcarrier at the same time. Thus, the statistical multiplexing is an efficient way for the access.

Applying the statistical multiplexing to OFDMA systems could improve the user capacity of the systems. Here the user capacity is defined as the maximum number of users supported by a BS with a basic data-rate. The RFH-OFDMA in [5] achieves higher user capacity than the traditional OFDMA systems when the channel activity is relatively low. However, the RFH-OFDMA induces unnecessary subcarrier collision as invoking the random frequency hopping pattern. When the channel activity is high, the user capacity of RFH-OFDMA is even lower than that of the traditional OFDMA.

We propose the MG-FH OFDMA that has much lower subcarrier collision probability by employing the deterministic hopping pattern and arranging the collisions properly. The subcarriers are utilized sufficiently through the application of the grouping scheme. The Latin Square hopping scheme and user index updating scheme randomize the subcarrier collisions among different users. Simulations show that the proposed MG-FH OFDMA is more effective upon the improvement of the user capacity than the RHF-OFDMA system.

The rest of the paper is organized as follows. Section II introduces the MG-FH OFDMA system models. The frequency hopping strategy of MG-FH OFDMA is described in section III Section IV analyses subcarrier collision and user capacity. The simulation results are given in section $\mathrm{V}$ and section VI concludes the paper.

\section{SYSTEM MODEL}

The downlink system of the proposed MG-FH OFDMA is presented as Fig.1. MG-FH OFDMA integrates the multi-group frequency hopping scheme into the OFDMA system. As shown in Fig.1, users are divided into several groups. Statistical multiplexing is applied among these different groups, while

This research has been supported by the Program for New Century Excellent Talents in University of China. 
deterministic frequency hopping pattern is employed within each group. The same subcarrier can not be allocated to different users in the same group, but users in different groups can share the same subcarrier. The frequency hopping is completed in every symbol duration.

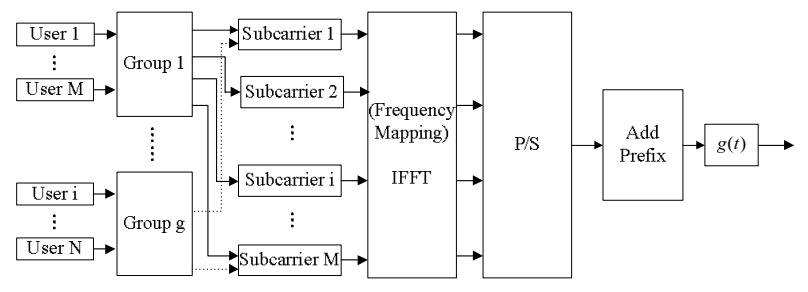

Figure 1. Transmitter diagram of MG-FH OFDMA downlink

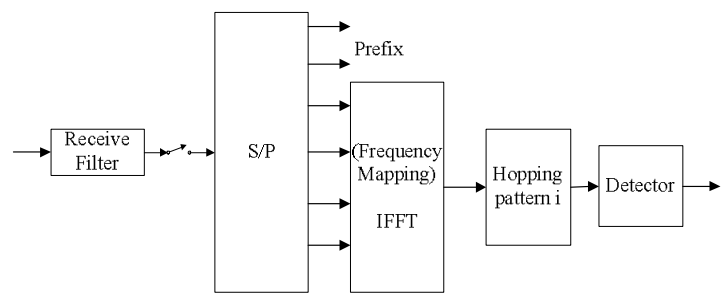

Figure 2. Receiver diagram of MG-FH OFDMA downlink

The user, who keeps up with the data service but does not need data transmission, releases the subcarrier and only traces the hopping pattern. When the user requires data transmission, BS firstly checks whether somebody else wants to use the corresponding subcarrier or not at the same time. If the answer is no, the data are mapped onto the subcarrier and transmitted. On the contrary, if others want to use the same subcarrier at the moment, subcarrier collision will happen. Then the BS compares user data experiencing subcarrier collisions and determines whether all the data are the same or not. If all corresponding data symbols are the same, the collision needs not be controlled, and all the colliding symbols of the different users are transmitted with one symbol energy. Otherwise, all the corresponding data symbols would not be transmitted during the symbol interval, which results in information loss. The lost information can be recovered by proper channel coding techniques.

\section{THE STRATEGY OF MULTI-GROUP OFDMA}

In the proposed MG-FH OFDMA systems, each user selects the subcarrier according to the multi-group hopping strategy that determines the frequency hopping pattern and the subcarrier collision probability. There are essentially three parts of the multi-group collision strategy: grouping scheme, deterministic frequency hopping scheme and user index updating scheme.

Suppose the number of users is $N$, and the number of available subcarriers is $M$. Let $\bar{v}$ denotes the channel active rate. The frequency hopping cycle $T_{M G}$ depends on the deterministic hopping scheme. Each user is allocated a specific user index $x, x \in(0,1 \cdots \cdots N-1)$, which affects its hopping pattern and does not change during a frequency hopping cycle. The OFDM symbol duration is denoted as $T_{s}$.

Details of the three parts are described in the following.

\section{A. Grouping scheme}

The grouping scheme ensures that all of the subcarriers are utilized sufficiently. All users are divided into $G=\lceil N / M\rceil$ groups. The number of group, that user with the index $x$ in a frequency hopping cycle belongs to, is obtained by

$$
g(x)=\lceil(x+1) / M\rceil .
$$

The frequency hopping index in the corresponding group is

$$
k(x)=x-(g(x)-1) \cdot M .
$$

Statistical multiplexing is applied between the different groups. It is obvious that the more users share the same subcarrier, the higher subcarrier collision probability will be. The RFH-OFDMA invokes random hopping strategy. Due to the properties of the random hopping strategy, a subcarrier may be allocated to numerous users while others may even not be used. It results in many unnecessary subcarrier collisions. The proposed MG-FH OFDMA employs the grouping scheme to avoid this problem. Grouping scheme averages users to different groups and utilizes the subcarrier sufficiently. Thus, the subcarrier collision probability is reduced effectively.

\section{B. Deterministic Hopping Scheme}

The deterministic hopping scheme is applied among the users in the same group. As mentioned above, the deterministic hopping scheme should guarantee orthogonal intra-group multiple access and randomize the inter-group subcarrier collision, also get the frequency diversity gain as large as possible. The scheme of orthogonal Latin Squares is invoked into frequency hopping strategy for its good properties [4].

Let $M$ be a prime number. Based on the Latin Squarebased hopping scheme, the subcarrier allocated to the user with the index $x$ in the OFDM symbol interval $j$ is expressed as

$$
s_{j}^{k(x), g(x)} \triangleq\left((k(x)-j) \alpha_{g(x)}^{-1}\right) \bmod M, \alpha_{g(x)}=1,2 \cdots M,
$$

during a hopping cycle according to (1) and (2), where $\alpha_{g(x)}$ is the hopping factor that effects the hopping patterns of the users. Each group is allocated with a specific hopping factor, as $\alpha_{g(x)} \neq \alpha_{g(y)}$, if $\mathrm{g}(x) \neq g(y)$. According to the properties of the Latin Square hopping scheme, the patterns in each group are orthogonal and collide only once with the patterns of the other group. The frequency hopping cycle of the multi-group becomes $T_{M G}=M \cdot T_{s}$.

\section{User Index Updating Scheme}

If the number of users is larger than that of available subcarriers, the last group as the Gth group contains $N+M-M \cdot G$ users while the other groups contain $M$ users. The subcarrier collision probability for the user in the Gth group is 


$$
P_{c, G}=1-(1-\bar{v})^{G-1} .
$$

The subcarrier collision probability for the user in the other $G-1$ groups is

$P_{c, \text { normal }}=1-\frac{M \cdot G-N}{M}(1-\bar{v})^{G-2}-\frac{N+M-M \cdot G}{M}(1-\bar{v})^{G-1}$.

It is obvious that $P_{c, \text { normal }} \leq P_{c, g}$, which results in the unfairness for the users in the Gth group. In order to avoid this problem, user index updating scheme is employed. The user index is expected to be changed in every multi-group frequency hopping cycle $T_{M G}$. The index $x(t)$ for the user updates to be

$$
x\left(t+T_{M G}\right)=(x(t)+N+M-M G) \bmod (N) .
$$

With this mechanism, every user has the same chance to be the member of the Gth group. It is also helpful to randomize the subcarrier collisions.

\section{Summerize}

As described above, the grouping scheme assures sufficient uses of the subcarriers, the Latin Square hopping scheme and user index updating scheme randomize the subcarrier collisions among different users. When a mobile terminal accesses to the MG-FH OFDMA system, it only needs to get his user index and the number of users at the beginning of a frequency hopping cycle. Then it can calculate its hopping pattern easily. Suppose the user is assigned with user index $x(0)$ at the beginning, the user index at time $t$ will change into

$$
x(t)=\left\{x(0)+\left\lfloor t / T_{M G}\right\rfloor(N+M-M G)\right\} \bmod (N) .
$$

Then the hopping pattern is easily obtained according to (1), (2) and (3).

\section{SUBCARRIER COLLISION PROBABILITY AND USER CAPACITY}

\section{A. Subcarrier Collision Probability}

Subcarrier collision happens among the users in different groups in the proposed MG-FH OFDMA system. When the number of users is less than the number of available subcarriers (e. g. $N \leq M$ ), there is only one group. The users do not suffer subcarriers collision as their hopping patterns are orthogonal. Then the subcarrier collision probability in this case is zero.

If the number of users is larger than that of available subcarriers, the subcarrier collision probability of the Gth group and the other groups should be considered respectively. Then, the subcarrier collision probability under this case is

$$
P_{c}=\frac{N-M \cdot(G-1)}{N} P_{c, G}+\frac{M \cdot(G-1)}{N} P_{c, \text { normal }} .
$$

According to (4) and (5), the subcarrier collision probability of the users with the MGC-FH OFDMA can be expressed as

$$
P_{c, M G}= \begin{cases}0 & \text { when } N \leq M \\ 1-\frac{(N+M-M \cdot G)}{N}(1-\bar{v})^{g-1}- & \\ \frac{(G-1)(M \cdot G-N)}{N}(1-\bar{v})^{G-2} & \text { when } N>M\end{cases}
$$

The subcarrier collision probability of RFH-OFDMA $P_{c, R F H}$ is calculated in [5]. Since it is difficult to compare $P_{c, M G}$ with $P_{c, R F H}$ mathematically, simulations is explored to analyze the two probabilities intuitively in the next section.

\section{B User Capacity}

The user capacity is limited by intra-cell capacity or the other cell interference. Intra-cell capacity denotes the maximum number of users accommodated for the OFDMA system. It is known that the user capacity is limited by OCI if the required $E_{b} / N_{0}$ is high (interference limited situation). Contrarily, the user capacity is limited by the intra-cell capacity if the required $E_{b} / N_{0}$ is low (resource limited situation) [9], [10].

The intra-cell capacity of the MG-FH OFDMA system for a given subcarrier collision probability is written as:

$$
C_{M G}=\frac{M(\bar{G}-1)(1-\bar{v})^{G-2}(\bar{G}+\bar{v}-1)}{1-P_{c, \text { max }}+(1-\bar{v})^{G-2}(\bar{G}+\bar{v}-2)},
$$

where $\bar{G}=1+\left\lceil\log _{(1-\bar{v})}\left(1-P_{c, \max }\right)\right\rceil$. The intra-cell capacity improves as the maximum of acceptable collision probability $P_{c, \text { max }}$ increases. It should be reminded that the transmit power should be augmented to mitigate the degradation of performance caused by subcarrier collisions. If $P_{c}$ increases, the additional energy $\Delta P$ need to be higher in order to satisfy the given FER requirement. However, the increase of $\Delta P$ might cause the aggravation of other cell interference.

In this paper, the cellular system whose frequency reuse factor equals to 3 is considered. The lower bound of the user capacity due to OCI in the cellular system is approximately expressed as [5]:

$$
C_{O C I} \simeq \frac{\beta q^{\alpha} M}{6 \mu \lambda R^{F E C}\left[\bar{v} \cdot\left\{\left(\frac{E_{b}}{I_{0}}\right)_{\text {required }}+\Delta P\right\}\right]},
$$

where $\left(E_{b} / I_{0}\right)_{\text {required }}$ denotes the required $E_{b} / N_{0}$ at the receiver for a specific data rate. $R^{F E C}$ and $\mu$ relate to the channel code rate and the modulation order respectively. $q$ is obtained by

$$
q=\frac{D}{r}
$$


where $D$ denotes the distance between the home-cell BS and the co-channel cell BS in the first tier, while $r$ denotes the cell radius. $\lambda$ is the sector antenna factor (e.g. $\lambda=1$ for a omniantenna case and $\lambda=1 / 3$ for 3 -sector antenna case). The value of $\beta$ is determined by the frequency hopping factor. Since the frequency reuse factor is set to 3 in this paper, it is calculated as

$$
\beta=\frac{1}{1+\left(\frac{1}{\sqrt{3}}\right)^{\alpha}+\left(\frac{1}{\sqrt{4}}\right)^{\alpha}+\left(\frac{1}{\sqrt{7}}\right)^{\alpha}+\cdots},
$$

The equation (11) indicates that the user capacity determined by the OCI is decreased as the additional power $\Delta P$ increases. Thus, the user capacity is the trade off between the intra-cell capacity and the OCI capacity. Since the value of intra-cell capacity and the OCI capacity are both affected by $P c$, the user capacity of the proposed MG-FH OFDMA system is expressed as

$$
C_{\text {celluar }}=\max _{P_{c} \leq P_{c, \max }}\left\{\min \left\{C_{O C I}, C_{M G}\right\}\right\} .
$$

The BS compares the tradeoffs of intra-cell capacity and the OCI capacity with different collision probabilities, and takes the maximum value as the user capacity of the system.

\section{SimUlation RESUlts}

In this section, numerical results are provided for a system with parameters listed in TABLE I. Cellular system which frequency reuse factor equals to 3 is considered. A multipath fading channel is taken into account with the RMS value of delay spread is $5 \mu \mathrm{s}$. All the used subcarriers are divided into 15 FH-hopping clusters and every user take 1 subcarrier in each cluster. In other words, each cluster consists of 53 subcarriers. The user may get his original user indexes in each cluster independently. In this case, the number of available subcarriers is 53. QPSK is employed as the modulation of the data and turbo codes (a code rate of 1/3) in 3GPP specifications with an MAP decoder is applied.

TABLE I. SYSTEM PARAMETERS

\begin{tabular}{|c|c|}
\hline Center frequency & $1.9 \mathrm{GHZ}$ \\
\hline Bandwidth & $\sim 5 \mathrm{MHZ}$ \\
\hline FFT size & 1024 \\
\hline Number of used subcarriers, $N_{C}$ & 795 \\
\hline OFDM symbol duration, $T_{s}$ & $230 \mu \mathrm{s}$ \\
\hline Tone spacing, $1 / T$ & $11.25 \mathrm{KHZ}$ \\
\hline
\end{tabular}

The required $E_{b} / N_{0}$ for an FER of 0.01 is approximately $1.35 \mathrm{~dB}$ when no subcarrier collision exists. When the subcarrier collisions occur, four grades of subcarrier collision and the relative additional energy for 0.01 FER are presented in TABLE II. in order to simplify the calculation of user capacities [5].

The additional energy may be varying for different channel codes or simulation parameters. But it will not affect the comparison of user capacities. Since the error correction capability of the channel code is limited, there must be an upper bound of subcarrier collision probability. As shown above, 0.4 is accepted to be the maximum subcarrier collision probability in this paper.

\begin{tabular}{|c|c|}
\hline subcarrier collision probability $\left(P_{c}\right)$ & $\Delta P(\mathrm{~dB}$ (integer) $)$ \\
\hline 0 & 0 \\
\hline $0 \sim 0.2$ & 1 \\
\hline $0.2 \sim 0.3$ & 2 \\
\hline $0.3 \sim 0.4$ & 3 \\
\hline
\end{tabular}

TABLE II. $\quad P_{c}$ AND CORRESPONDING $\Delta P$

The subcarrier collision probabilities of MG-FH OFDMA and the RFH-OFDMA are shown in Figure 3It is obvious that the MGC-FH OFDMA suffers lower subcarrier collision probability than the RFH-OFDMA in the acceptable collision probability area. When there are too many users, the subcarrier collision probabilities of both two systems become too high to mitigate. It will never be allowed to accommodate so many users. Then the comparison in this case would not to be considered.

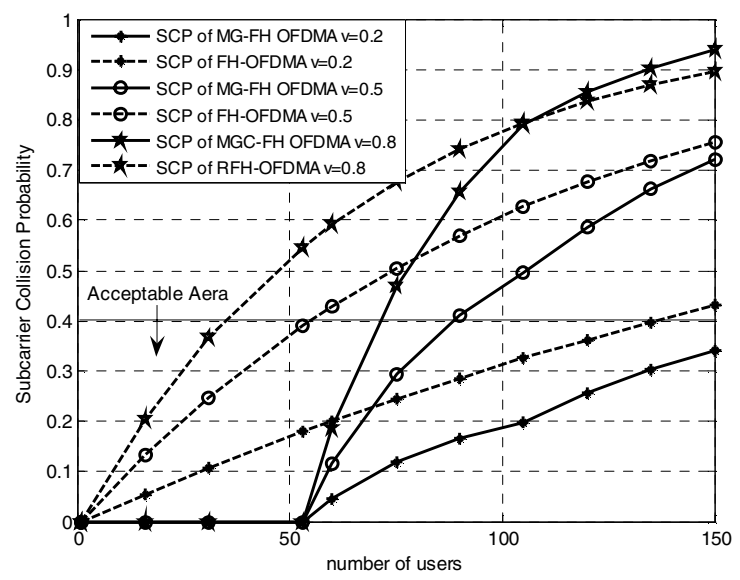

Figure 3. Comparision of the subcarrier collision probability (SCP)

User capacity for varying subcarrier collision probabilities is shown in Figure 4The channel activity is set to be 0.5 . When the required $E_{b} / N_{0}$ equals to $1.35 \mathrm{~dB}$, both the $\mathrm{MG}-\mathrm{FH}$ OFDMA system and RFH-OFDMA system is in the resource limited situation. Thus the user capacity of the two systems equals to their intra-cell user capacity. It is easily to see that the user capacities of MG-FH OFDMA system and RFH-OFDMA system are 72 and 54 respectively in this case. When the required $E_{b} / N_{0}$ equals to $6 \mathrm{~dB}$, the situation is more complex. In the omni-antenna cellular systems, the OFDMA systems are in the interference limited situation as shown in Figure 4(a). According to (12), the RFH-OFDMA gets its user capacity that equals to the maximum tradeoff between the intra-cell capacity and the OCI capacity with different collision probabilities. It is obvious that the maximum tradeoff is gotten when $P c=0.3$ and equal to $C_{o c i}$. Thus, the user capacity of RFH-OFDMA is 31. Meanwhile, the MG-FH OFDMA obtains the user capacity as 50 which is equal to $C_{o c i}$ when $P c=0$. Figure 4(b) shows 
the user capacities of the OFDMA systems in the 3-sector cellular systems. In this case, the user capacities of MG-FH OFDMA system and RFH-OFDMA system are 72 and 54 respectively, both equal to the intra-cell capacities when $P c=0.4$. To sum up, the MG-FH OFDMA gets higher user capacity than the RFH-OFDMA when the channel activity is 0.5 .

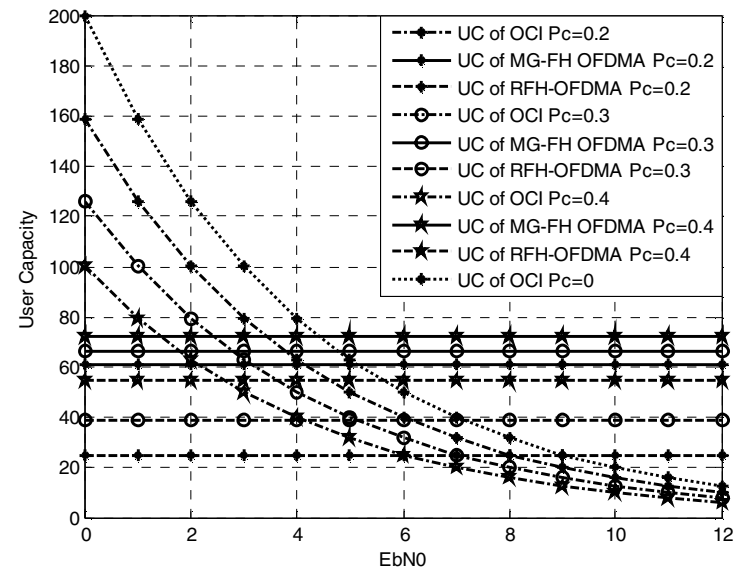

(a) $v=0.5, \quad \lambda=1$

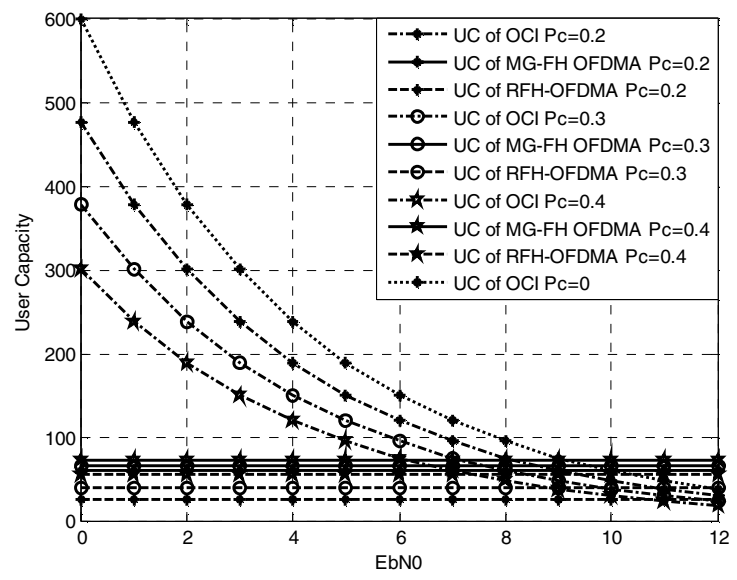

(b) $v=0.5, \lambda=1 / 3$

Figure 4. User Capacity (UC) for varying subcarrier collision probability

The simulations of user capacity for the OFDMA systems when the channel activity equals to $0.2,0.8$ and 1 are completed respectively. As lack of space, the results of these simulations are listed in TABLE III. It is obvious that proposed MG-FH OFDMA can also improve the user capacity compared with the traditional OFDMA systems and shows greater advantage than the RFH-OFMDA system.

\section{CONCLUSION}

In this paper, we propose the MG-FH OFDMA based on the statistical multiplexing. The MG-FH OFDMA divides the users into several groups and applies statistical multiplexing to different groups. By invoking the Latin Square hopping scheme and user index updating scheme, the subcarrier collisions among the users are randomized. The user capacity is analyzed by considering both the intra-cell capacity and other cell interference. Simulations show that the proposed MG-FH OFDMA obtains higher user capacity than that of the RFHOFDMA system. Furthermore, MG-FH OFDMA can still accommodate more users than the conventional FH-OFDMA systems in the case of high channel activity.

TABLE III. USER CAPACITY.

\begin{tabular}{|c|c|c|c|c|c|c|}
\hline \multicolumn{7}{|c|}{$P_{c, \text { max }}=0.4,\left(E_{b} / I_{0}\right)_{\text {required }}=1.35 \mathrm{~dB}$} \\
\hline & \multicolumn{3}{|c|}{$\lambda=1$} & \multicolumn{3}{|c|}{$\lambda=1 / 3$} \\
\hline $\mathbf{v}$ & FH & $\begin{array}{c}\text { RFH- } \\
\text { OFDMA }\end{array}$ & $\begin{array}{l}\text { GM-FH } \\
\text { OFDMA }\end{array}$ & FH & $\begin{array}{c}\text { RFH- } \\
\text { OFDMA }\end{array}$ & $\begin{array}{l}\text { GM-FH } \\
\text { OFDMA }\end{array}$ \\
\hline 0.2 & 53 & 136 & 162 & 53 & 136 & 162 \\
\hline 0.5 & 53 & 54 & 72 & 53 & 54 & 72 \\
\hline 0.8 & 53 & 34 & 59 & 53 & 34 & 68 \\
\hline 1 & 53 & 27 & 58 & 53 & 27 & 66 \\
\hline \multicolumn{7}{|c|}{$P_{c, \text { max }}=0.4,\left(E_{b} / I_{0}\right)_{\text {required }}=6.0 \mathrm{~dB}$} \\
\hline & \multicolumn{3}{|c|}{$\lambda=1$} & \multicolumn{3}{|c|}{$\lambda=1 / 3$} \\
\hline $\mathbf{v}$ & FH & $\begin{array}{c}\text { RFH- } \\
\text { OFDMA }\end{array}$ & $\begin{array}{c}\text { GM-FH } \\
\text { OFDMA }\end{array}$ & FH & $\begin{array}{c}\text { RFH- } \\
\text { OFDMA }\end{array}$ & $\begin{array}{c}\text { GM-FH } \\
\text { OFDMA }\end{array}$ \\
\hline 0.2 & 53 & 79 & 99 & 53 & 136 & 162 \\
\hline 0.5 & 50 & 31 & 50 & 53 & 54 & 72 \\
\hline 0.8 & 31 & 19 & 31 & 53 & 34 & 59 \\
\hline 1 & 25 & 15 & 25 & 53 & 27 & 58 \\
\hline
\end{tabular}

\section{REFERENCES}

[1] Ahmad RS Bahai, Burton R. Saltzberg and Mustafa Ergen, Multi-Carrier Digital Communications: Theory and Applications of OFDM, second edition, Springer, 2004.

[2] Andrea Goldsmith, Wireless Communications, Cambridge University Press, 2005.

[3] T.Scholand, T.Faber, "Fast frequency hopping OFDM concept," Electronic letters, Vol.41, No.13, June. 2005

[4] Kostas Stamatiou and John G. Proakis, "A performance analysis of coded frequency-hopped OFDMA," IEEE Communication Society / WCNC, 2005

[5] Bang Chunl Jung and Dan Keun Sung, "Random FH-OFDMA system based on statistical multiplexing", IEEE Vehicular Technology Conference(VTC), 2005.

[6] Tolga Kurt, Hakan Delic, "On Symbol Collisions in FH-OFDMA," IEEE Vehicular Technology Conference (VTC), 2004.

[7] Lie-Liang Yang and Lajos Hanzo, "Residue Number System Assisted Fast Frequency-Hopped Synchronous Ultra-Wideband Spread-Spectrum Multiple-Access: A Design Alternative to Impulse Radio," IEEE Journal on Selected Areas in Communications, vol. 20, No. 9, Dec 2002

[8] Z Kostic, I Maric, X Wang, "Fundamentals of Dynamic Frequency Hopping in Cellular System," IEEE Journal on Selected Areas in Communication, Vol.19, No. 11, Nov. 2001.

[9] B. Sklar, Digital Communications, Prentice Hall, 2001

[10] Ivanov. K, Metzner. N, and Spring. G, "Frequency hopping spectral capacity enhancement of cellular networks," Spread Spectrum Techniques and Applications Proceedings, 1996. 